\title{
Cognitive outcome of traumatic brain injury in elderly patients
}

\author{
Prof.DR.Roshdy Abd Elaziz Elkhayat (1),Prof. DR.Momen Mohamed Almamoun(2),DR.Magda \\ Mohamed Ali (3),DR.Ahmed Mohamed EL Sayed Mohamed (2). \\ Neurosurgery Department, Faculty of Medicine, Sohag University (2) \\ And Assuit University (1), Lecturer of public health and community medicine Sohag \\ University (3).
}

\begin{abstract}
Objective: To assess the cognitive outcome of traumatic brain injury in elderly patients. Design: a prospective study.

Methodsthis study was conducted on 50 elderly patients exposed to TBI in sohag university hospital and 50 patients exposed to TBI but less than 65 years used as a control.

IntroductionTraumatic brain injury (TBI) is a significant problem in older adults, and is associated with changes in the brain that affect the cognition(Sapoznik et al 2006)

ResultsDuring the study period, 50 elderly patients with TBI were followed up for cognitive outcome, 12 patients $(24 \%)$ were associated with cognitive deficits.

Conclusion: TBI in elderly patients is associated with cognitive deficits post injury.

Key words:traumatic brain injury, elderly patients, cognitive outcome, geriatric trauma, prognosis.

\section{Introduction}

Trauma literature usually defines "elderly" as more than 65 years of age.(Patel HC, et al 2011)

structural changes in the brain include

Patient and methods:Arandomized study was conducted on 50elderly patients admitted to Sohag university hospital.
\end{abstract} neuronal reduction with a reduced volume of the frontal and medial temporal lobes resulting in difficulties with episodic memory and reasoning, also reduced neuronal plasticity leaves older adults with an exaggerated risk of cognitive decline (Kempermann et al., 2002)

Senathi-Raja et al. (2010) reported that elderly patients with traumatic brain injury were suffering of emotional and cognitive changes including slowness, memory loss and mood changes compared to those not exposed to TBI

\section{Aim of the study:}

The aim of this study is to assess cognitive outcome after traumatic brain injury in elderly patients above 65years.

Inclusion criteria: 50 patients with traumatic brain injury over 65 years old.

\section{Administrative design:}

- The study was approved by ethical committee of sohag faculty of medicine.

- Personal communications with responsible administrative authorities and written consent was obtained.

\section{The study includes:}

\section{1) Clinical assessment:}

-Thorough medical history taking,General examination, Neurological examination.

\section{2) Investigations:}

- Laboratory investigations (CBC, PT, PC, LFT and KFT).

- Head CT at admission time, if any deterioration occurs and on discharge. 
SOHAG MEDICAL JOURNAL Cognitive outcome of traumatic brain injury in elderly patients

- Brain MRI, MRA and MRV if indicated.

\section{3) Management:}

Patients were managed conservatively or surgically under general or local anesthesia.According to their type of brain injury.

\section{4) Follow up:}

All patients were followed up clinically during hospital stay and for one month after dischargeand assessingtheconscious level and presence or absence of cognitive deficit and radiographic investigations by Computed tomography and MRI if indicated.

\section{5) Statistical analysis:}

Data analyzed using STATA intercooled version 12.1. Quantitative data represented as mean, standard deviation, median and range. As the data was not normally MannWhitney test was used to compare two groups. Qualitative data was presented as number and percentage and compared using either Chi square test or fisher exact test. Graphs were produced by using Excel or STATA program. $P$ value was considered significant if it was less than 0.05 .

Results:cognitive outcome in the study group in 38 patients $(76 \%)$ were found free of cognitive deficits while 12 patients (24\%) had cognitive deficit, from them 9 patients (18) \% had dementia, 2 patients (4\%) had depression and 1 patient $(2 \%)$ had anxiety disorder.

Figure (1) shows cognitive follow-up in elderly patients exposed to TBI.

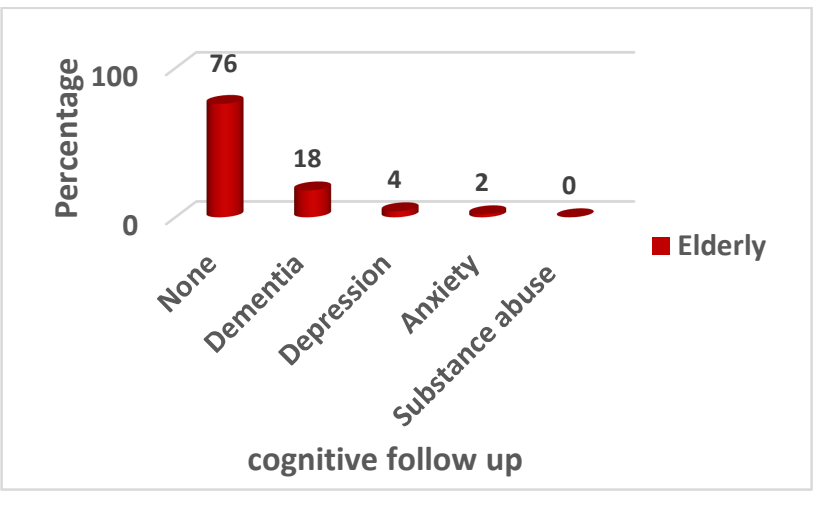

Table (1) shows cognitive follow-up in elderly patients exposed to TBI.

\begin{tabular}{|l||c||c||}
\hline Variable & $\begin{array}{c}\text { Elderly } \\
\text { N=50 }\end{array}$ & P value \\
\hline \hline Psychological follow-up & $38(76.00 \%)$ & \\
None & $9(18.00 \%)$ & $<0.0001$ \\
Dementia & $2(4.00 \%)$ & \\
Depression & $1(2.00 \%)$ & \\
Anxiety & 0 & \\
Substance abuse & & \\
\end{tabular}

\section{Discussion}

As regard cognitive outcome 50 elderly patients were examined 38 patients $(76 \%)$ did not have cognitive deficits while 12 patients $(24 \%)$ had cognitive deficit of them 9 patients (18)\% had dementia , 2 patients (4\%) had depression and 1 patient $(2 \%)$ had anxiety disorder which matches the results of a studyperformed by Wheelan-Goodinson et al (2010) comparing cognitive outcomes in patients exposed to TBI inone hundred patients (19-74 years) assessed at 6 months to five years post-injury for anxiety, depressive and substance abuse disorders. The authors found that those over 60 years had lower rates of anxiety disorder and drug abuse and higher rates of dementia and depression.

\section{$\underline{\text { Limitations }}$}

Many studies examining outcomes among TBI patients simply exclude participants that died during hospitalization or at follow-up. 
SOHAG MEDICAL JOURNAL Cognitive outcome of traumatic brain injury in elderly patients

Follow-up time after injury is short.

Small number of cases.

\section{References}

1- Kempermann, G., Gast, D., \& Gage, F. H. (2002). Neuroplasticity in old age: Sustained fivefold induction of hippocampal neurogenesis by long-term environmental enrichment. Annals of Neurology, 52(2), 135-143

2- Patel HC, Bouamra O, Woodford $\mathbf{M}$, et al (2010). Clinical article: mortality associated with severe head injury in the elderly. ActaNeurochir (Wien). (2010).

3- Sapoznik, S., Ivenshitz, M., \& Segal, M. (2006). Age-dependent glutamate induction of synaptic plasticity in cultured
ConclusionTBI is a leading cause of cognitive deficit in elderly patients

hippocampal neurons. Learning and Memory, 13(6), 719-727.

4- Senathi-Raja, D., Ponsford, J., \& Schonberger, M. (2010). The association of age and time postinjury with long-term emotional outcome following traumatic brain injury. Journal of Head Trauma and Rehabilitation, 25(5), 330-338.

5- Wheelen-Goodinson, R., Ponsford, J. L., Schonberger, M., \& Johnston, L. (2010). Predictors of psychiatric disorders following traumatic brain injury. Journal of Head Trauma and Rehabilitation, 25(5),

320-3. 